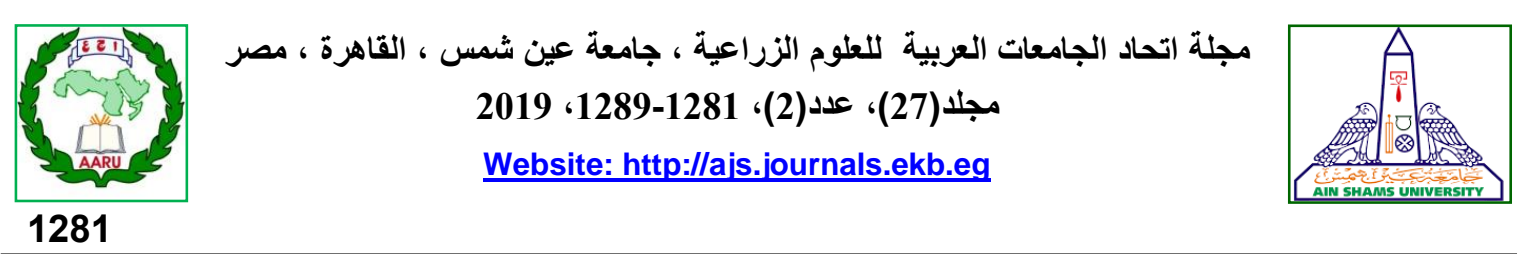

التنبؤ بسلوك أهم المتغيرات الاقتصادية للأعلاف المصنعة من اعلاف الماثية وإلدواجن

[107]

$$
\begin{aligned}
& \text { محمود صلاح سالم " ـ ثناء النوبي أحمد سليم- ايمان فريد أمين قادوس }
\end{aligned}
$$

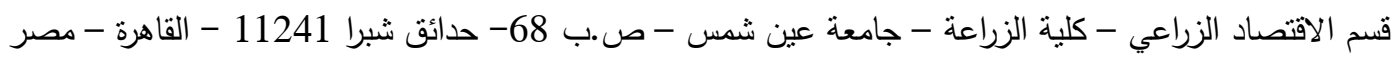

*Corresponding author: engmahmoudsalah8@gmail.com

Received 22 April, 2019 Accepted 10 June, 2019

الميزان التجاري وبصفة خاصة مع ارتفاع سعر الدولار .

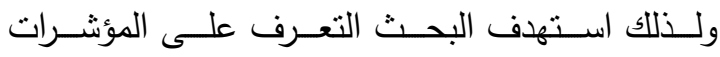

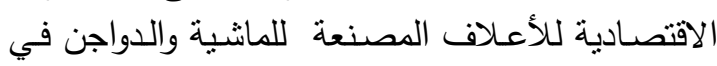

مصر

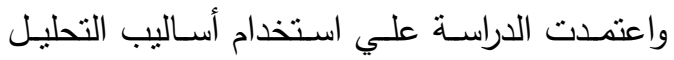

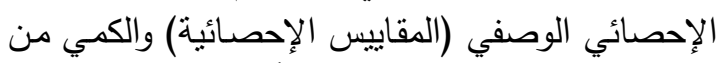

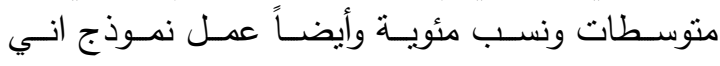

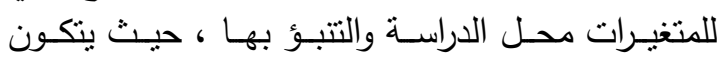

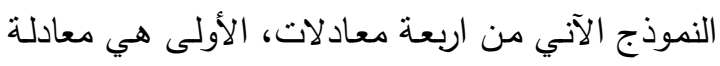

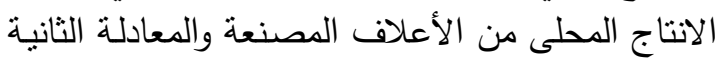

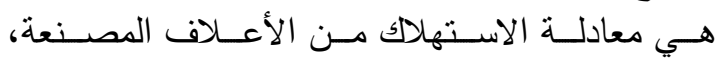

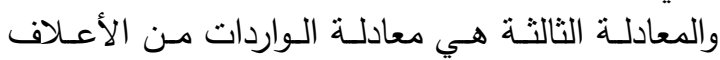

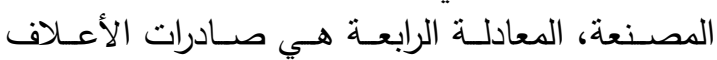

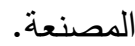

الكلمـات الدالـة: الأعلاف المصنعة، اعلاف المانشية، اعلاف الدواجن، النموذج الآني

\section{مقدمـة}

يتطلب النهوض بالإنتاج الحيواني توفر مستلزمات

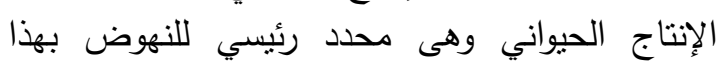
القطاع الهام، حيث بلغت قيثة الإن الإنتاج الحيواني 144.9 مليار جنيه في عام 2016-2017 الإن بنسبة

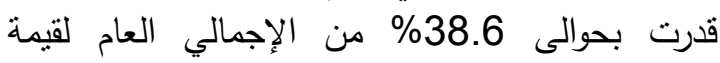
الإنتاج الزراعي البالغ نحو 38.6 374.9 مليار جنيه في الإلي نفس العام وبلغت قيمة لحوم الدواجن حوالئ حوالى 32.8 مليار جنيه عام 2016-2017 وبلغ قيمة البيض نحو لئي

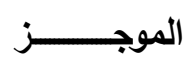

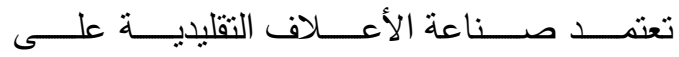
مواد العلف الخام المركزة وقد تكون هذه المواد غنية في الطي

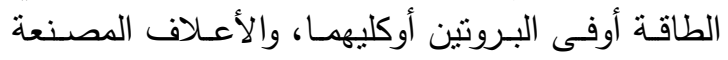

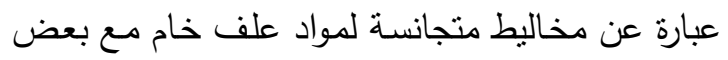

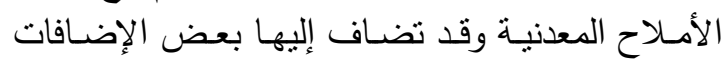

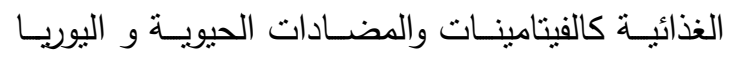

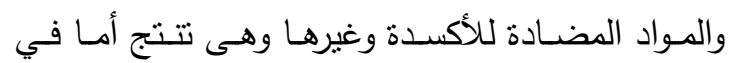

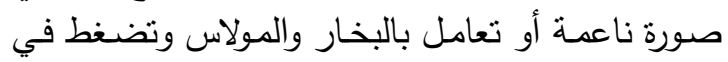

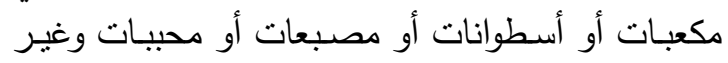

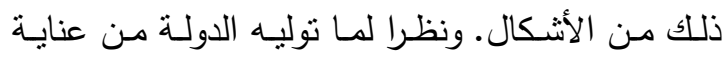

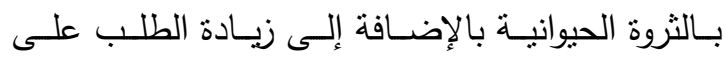

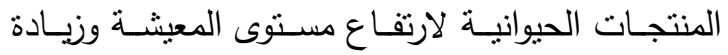

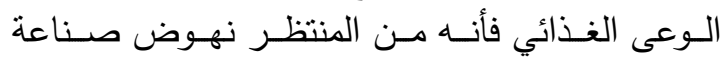
الأعلاف خلال السنوات القليلة القادمة.

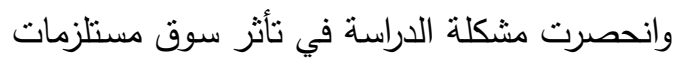

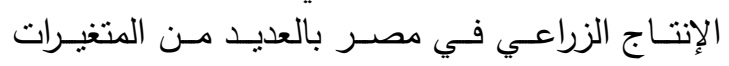

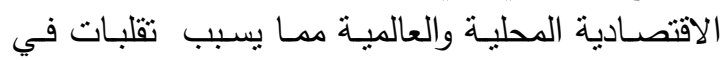

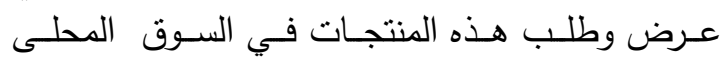

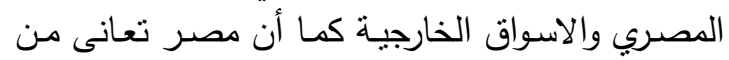

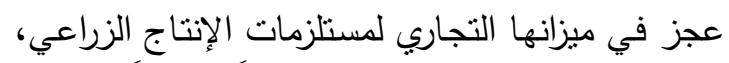

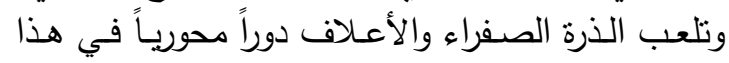
العجز ، وكذلك انخفاض كمية الأعلاف المركزة وأهمها

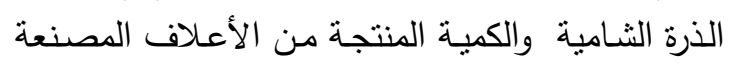

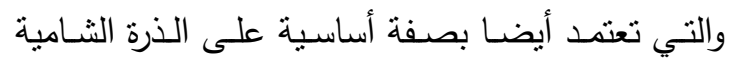
خاصاً علف الدواجن الذى يشكل الذرة الثامية الصفراء

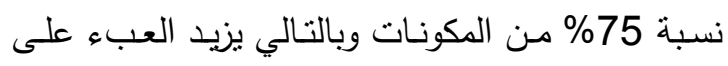


للمتغيـرات محـل الدراسـة والتتبـؤ بهـا ، حيـث يتكـون

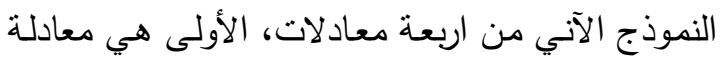

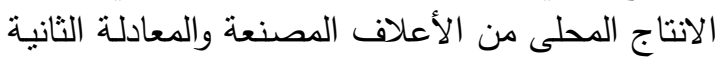

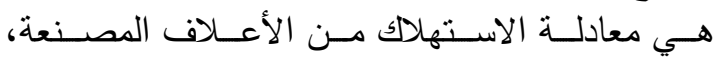

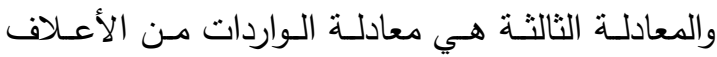

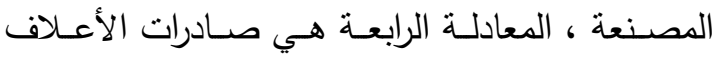
المصنعة. قد اعتمد البحث لتحقيق أهدافه على البيانات

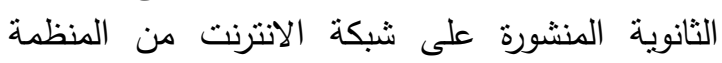

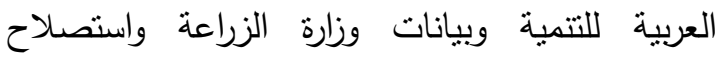

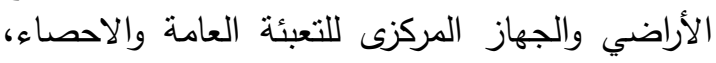

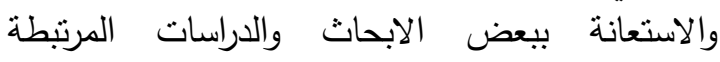

$$
\text { بموضوع البحث. }
$$

نتائج البحث

\section{توصيف النموذج}

لداسة تأثثر مجموعة المتغيرات التي تؤثنر على

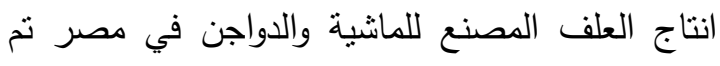

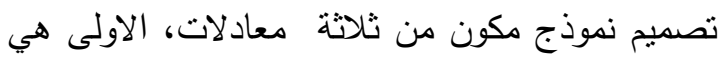

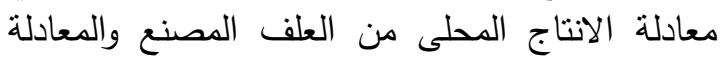

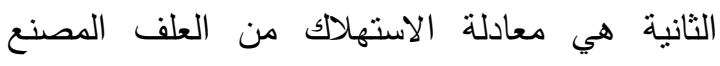
المستخدم كعلف والمعادلة الثالثة هي معادلة الواردات من العلف المصنع بينما المعادلة الرابعة معادلة المعادلة الرادئة

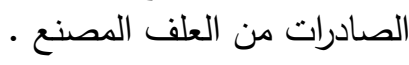

\section{1- معادلة الانتاج من العلف المصنع:}

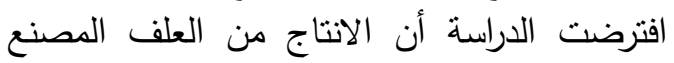

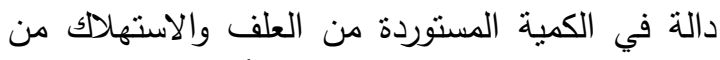

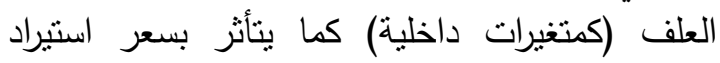

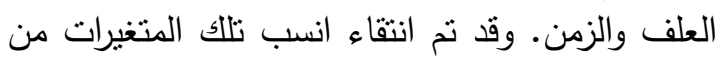
الناحية الاحصائية والاقتصادية والقياسية ويمكن تمثيل

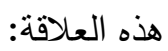

12 مليار جنيه في نفس العام بنسبة قدرت بنحو

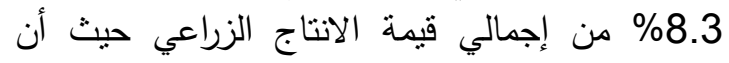

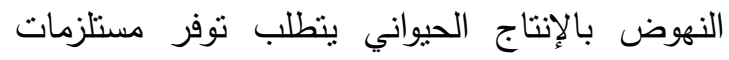
الإنتاج الحيواني وهى محدد رئيسي للنهوض بلإن بهذاج

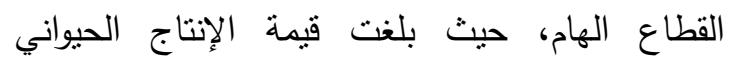

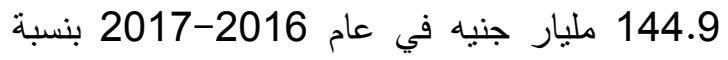

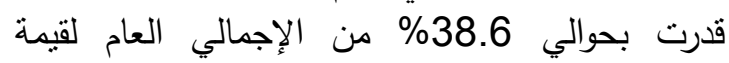

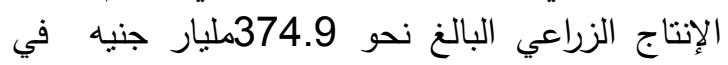
نفس العام وبلغت قيمة لحوم الدواجن حوالى 32.8

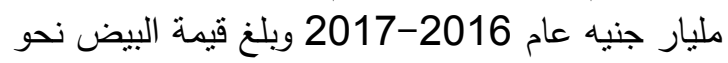
12 مليار جنيه في نفس العام بنسبة قدرت بنح 8.3 من إجمالي قيمة الانتاج الزراعي.

\section{مشكلة الدراسة}

تكمن مشكلة الدراسة في تأثز سوق مستلزمات

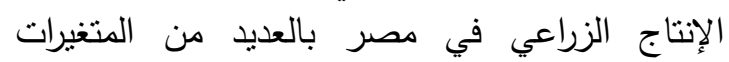

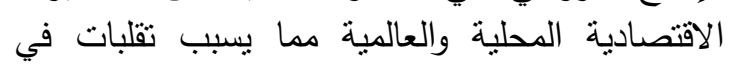

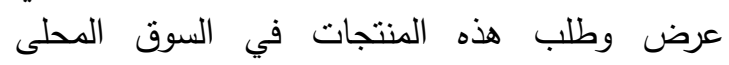

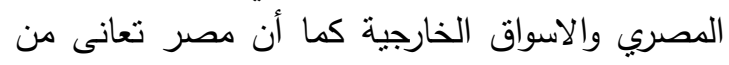

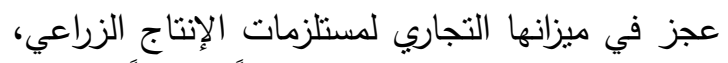

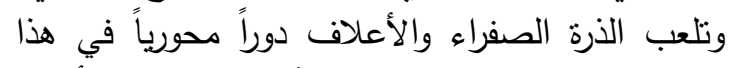
العجز ، وكذلك انخفاض كمية الأعلاف المركزة وأهمها

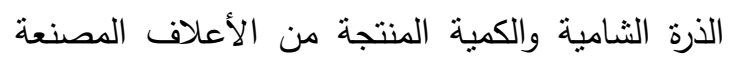

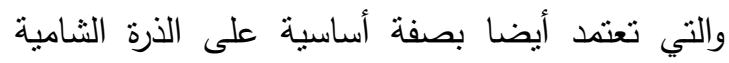

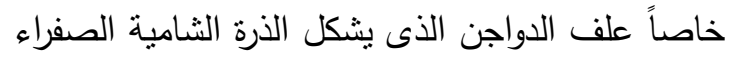

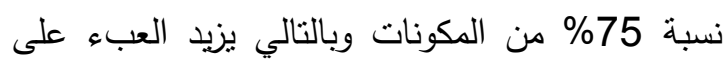
الميزان التجاري وبصفة خاصة مع ارتفاع سعر الدولار .

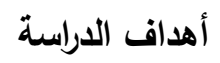

ولذلك استهدف البحث التعرف على المؤشرات

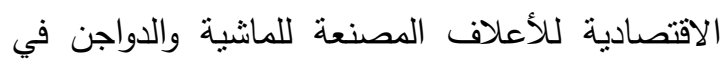

\section{$Y_{1 T}=F\left(y_{2 T} \cdot X_{2 T} \cdot y_{3 T} \cdot T\right)$}

الطريقة البحثية ومصادر البيانات

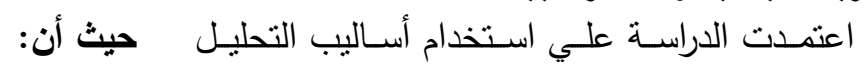

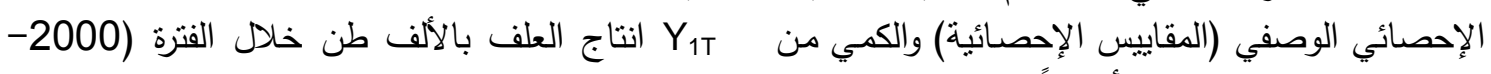

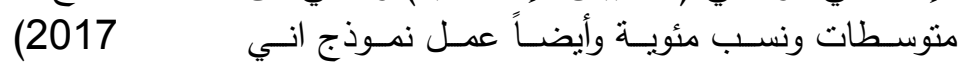




\section{3}

التتبؤ بسلوك أهم المتغيرات الاقتصادية للأعلاف المصنعة من اعلاف الماشية والدواجن

\section{$Y_{3 T}=F\left(y_{1 T} \times X_{1 T} \cdot X_{2 T} \cdot X_{4 T} \cdot X_{5 T} \cdot y_{2 T}\right)$}

حيث أن:

الواردات من العلف بالألف طن خلا الفترة

(2017-2000)

الانتاج من العلف بالألف طن خلال الفترة

(2017-2000)

الاستهلاك من العلف بالألف طن خلال الفترة

(2017-2000)

سعر استيراد العلف بالجنيه/ طن خلال الفترة X2T (2017-2000)

سعر العلف المحلى جنيه/ طن خلا الفترة (2017-2000)

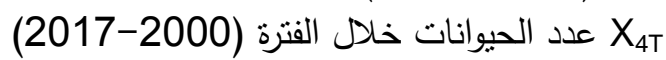
سعر صرف الدولار بالجنيه المصري خلال الفترة (2017-2000)

\section{4- معادلة الصادرات من العلف المصنع}

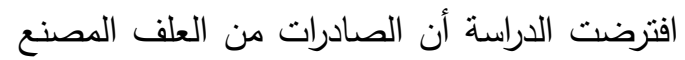
دالة في كمية الانتاج من العلف (كمتغيرات داخلية) وسعر تصدير العلف وسعر صرف الأنتاج من الدولار بالجنيه المصري وقد تم انتقاء أنسب تلإك المتغيرات من الناحية الاحصائية والاقتصادية والقياسية ويمكن تمثيل هذه النياء العلاقة: - الاعة

\section{$Y_{4 T}=F\left(Y_{2 T} ، Y_{1 T} 6 X_{5 T} \cdot X_{3 T}\right)$}

$$
\text { حيث أن: }
$$

الصادرات من العلف بالألف طن خلال الفترة (2017-2000)

سعر استيراد العلف بالجنيه/ طن خلال الفترة (2017-2000)

الانتاج من العلف بألف طن خلا الفترة (2017-2000)

سعر تصدير العلف بالجنيه/ طن خلال الفترة (2017-2000)

سعر صرف الدولار بالجنيه المصري خلال الفترة XT

(2017-2000)
الاستهالك من العلف بالألف طن خلال الفترة

(2017-2000)

الواردات من العلف بالألف طن خلال الفترة YT

(2017-2000)

X2T

(18.

3، 2، الزمن T T

2- معادلة الاستهلاك من العلف

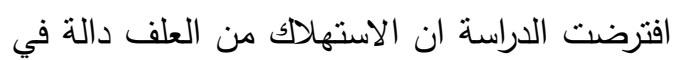

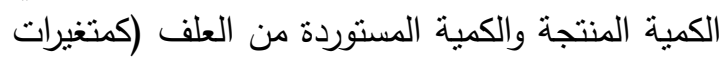

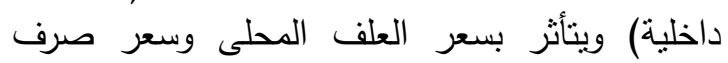
الدولار بالجنيه المصري وعدد الحيوانات وقد نم انتقاء

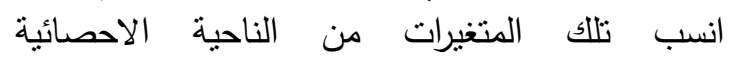
والاقتصادية و القياسية و يمكن تمثيل هذه العلاقة:

\section{$Y_{2 T}=F\left(y_{1 \top} \cdot X_{1 \top} \cdot x_{4 \top} \cdot X_{5 T} \cdot y_{3 T}\right)$}

حيث أن:

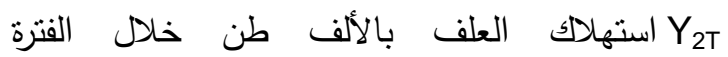
(2017-2000)

الانتاج من العلف بالألف طن خلا الفترة (2017-2000)

الواردات من العلف بالألف طن خلال الفترة

(2017-2000)

سعر العلف المحلى بالجنيه خلا الفترة X1T

(2017-2000)

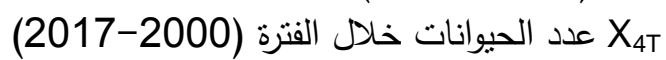
سعر صرف الدولار بالجنيه المصري خلال الفترة XT

(2017-2000)

3- معادلة الواردات من العلف المصنع:

افترضت الدراسة أن الواردات من العلف دالف دالة في في

الكمية المستهلكة والانتاج من العلف (كمتغيرات داخلية)

وسعر استيراد العلف وسعر صرف الاجناجن الدولار بالجنيه

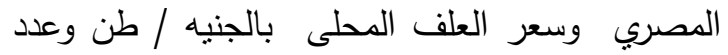

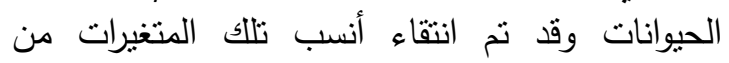

الناحية الاحصائية والاقتصادية والقياسية ويمكن تمثنيل

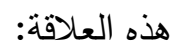


مع المنطق الاقتصادي وعكسي مع الواردات بقدار

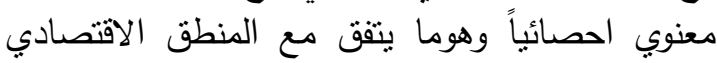
حيث أثنارت النتائج أن الكمية المنتجة من الأعلاف

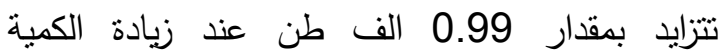
المستهلكة بالألف طن، وتتناقص كمية الانتاج من

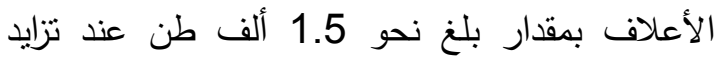

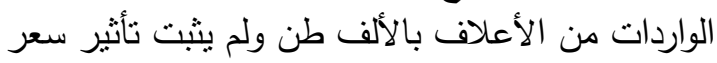

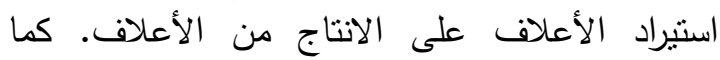

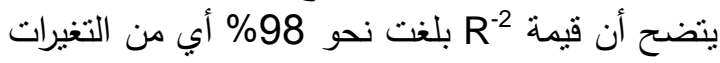
التي تحدث في الكمية المنتجة من الأعلاف ترجع التيرات

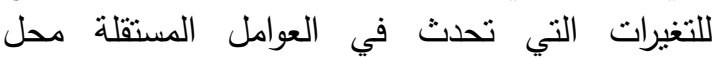
الدراسة.كما ثبتت معنوية النموذج المستخدم من خلال

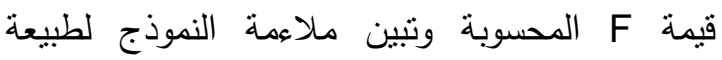
البيانات الاحصائية للظاهرة موضع الدراسة . $\mathrm{Y}_{1}=28+0.99 \mathrm{y}_{2}-1.5 \mathrm{y}_{3}+0.01 \mathrm{x}_{2}$

$$
\text { (0.4) }(25)^{* *}(6.8)^{\star *}(1.78)
$$

$\mathrm{R}^{-2}=0.98 \quad \mathrm{~F}=269.5$

$Y_{2}=701+0.9 y_{1}-0.03 x_{4}+1.62 y_{3}$

$$
(1.99)^{\star}(13.6)^{\star *}(2.2)^{\star} \quad(7.7)^{\star *}
$$

$R^{-2}=0.98 \quad F=269.5$

$Y_{3}=415-0.52 y_{1}+0.58 y_{2}$

$$
\text { (1.5) }(5.7)^{\star \star}(7.6)^{\star \star}
$$

$R^{-2}=0.72 \quad F=30.9$

$Y_{4}=11-1.49 x_{5}+0.001 x_{3}$

$$
\text { (1.63) (2.2) (6.6) }
$$

$R^{-2}=0.78 \quad F=19.5$ المصدر : جدول (1)، (2) بالدراسة .

2- معادلة استهلاك الأعلاف المصنعة في مصر

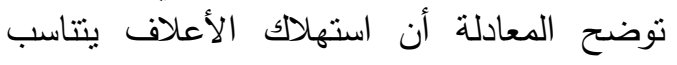

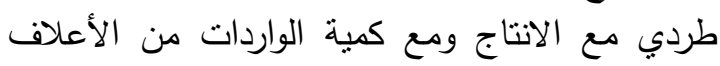

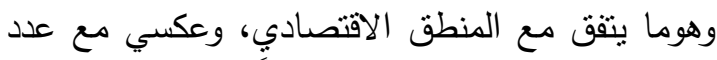

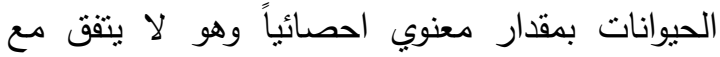

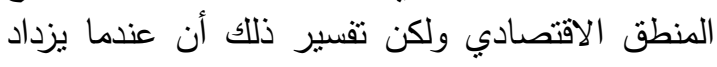
عدد الحيوانات يزداد الطلب على الأعلاف ولكن لأل الأن

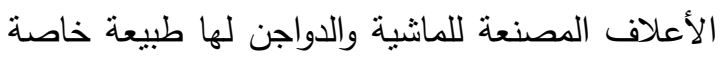

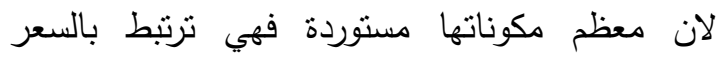
العالمي للمكونات وبالتالي فهي تتوقف على على الكميات المستوردة من خامات الأعلاف وأهها الذرة الثامية الكيات

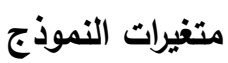

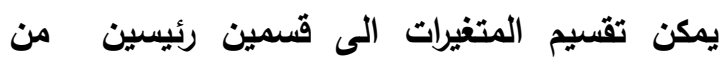

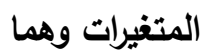

1- المتغيرات الاخلية Endogenous variables وهى المنغيرات التي ينم تقدير قيمها من داخل

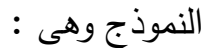
Y الانتاج من العلف بألف طن خلال الفنرة .2017-2000)

استهلالك الحيوانات من العلف بألف طن خلال الفترة (2000-2017)

Y الواردات من العلف بألف طن خلال الفترة (2017-2000)

الصادرات من العلف بألف طن خلال الفترة .2017-2000)

2- المتغيرات الخارجية Exogenous variables وهى المنغيرات التي بتم تقدير قيمها من خارج

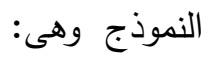
سعر العلف المحلى جنيه/ طن خلال الفترة X1T (2017-2000)

سعر استيراد العلف بالجنيه/ طن خلال الفترة X2T (2017-2000)

سعر تصدير العلف جنيه /طن خلال الفترة (2017-2000)

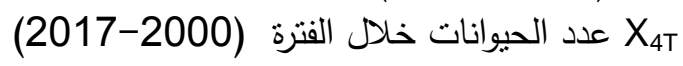
سعر صرف الدولار بالجنيه الدصري خلال الفترة XT X (2017-2000)

$(18$

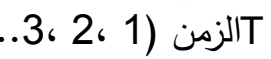

نتائج التقاير الإحصائي للنموذج الآني للعلف المصنع في مصر

تبين ان النموذج زائد التعريف النيف لذلك كانت طريقة المربعات الانبا ذات التبرئ المراحل الثلاث 3SLS هي أنسب الطرق المستخدمة 1- معادلة إنتاج الأعلاف المصنعة في مصر

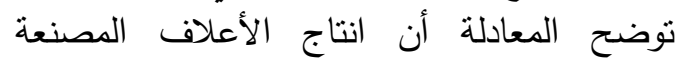
يتتاسب طردي مع الاستهلاك من الأعلاف وهوما يتفق الألقاف 


\section{التتبؤ بسلوك أهم المتغيرات الاقتصادية للأعلاف المصنعة من اعلاف الماثية والدواجن 1285}

3- معادلة الواردات من الأعلاف المصنعة في مصر توضح المعادلة أن الواردات من الأعلاف تنتاسب الأبلاف

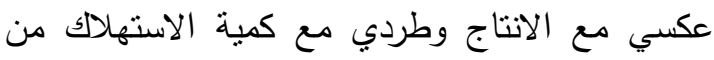

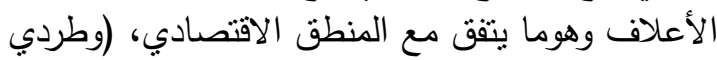

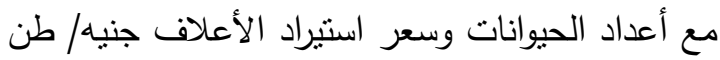

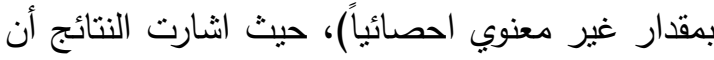

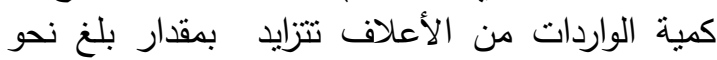

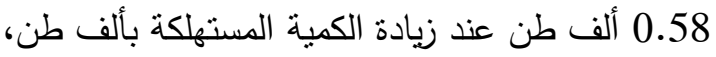

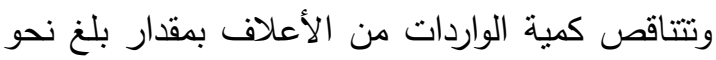

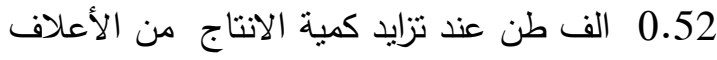
بألف طن وثثتت معنوية النموذج حيث أن أن كن التغيرات التي تحدث في كمية الواردات من الأعلاف ترجع للتغيرات الني تحدث في العوامل المستقلة محل

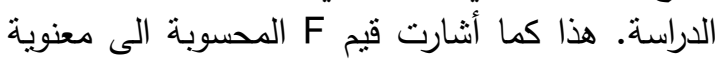

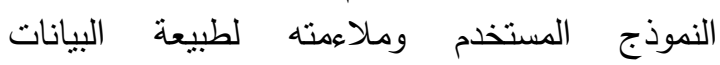
الاحصائية للظاهرة موضع الدراسة . ل

4- معادلة كمية الصادرات من الأعلاف المصنعة

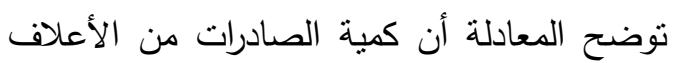

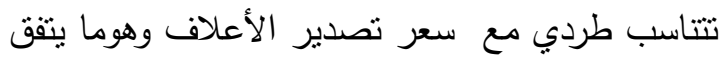

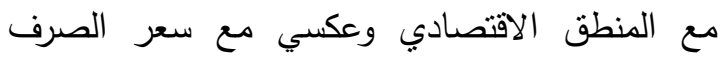

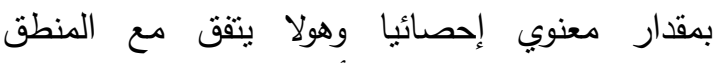

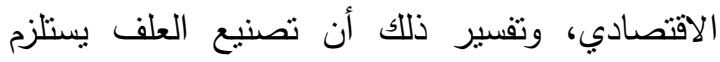

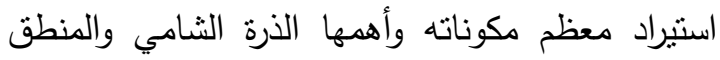

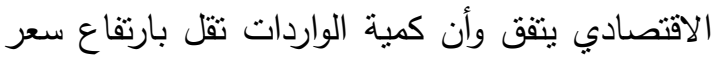
صرف الدولار وبالتالي يقل الانتان الواردات من باتل الأعلاف

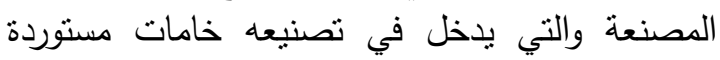
بنسبة 70\% وبالتالي يقل الانتاج لارتفاع سعر صرف فئل

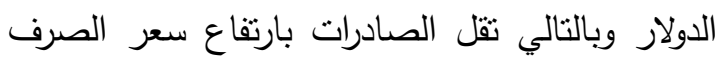

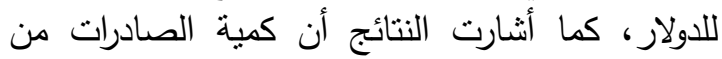

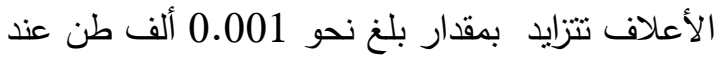

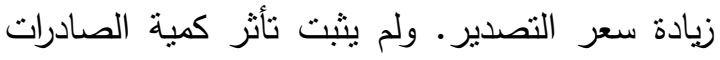

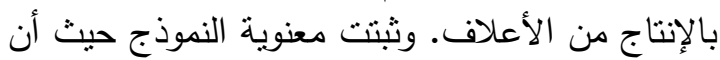
78 من التغيرات التي تحدث في كمية الصادرات من الأعلاف ترجع للتغيرات التي تحدث في في العوامل التئ المستقلة محل الدراسة.
كما سبق وأن تنين في النموذج الانى للذرة الثامية

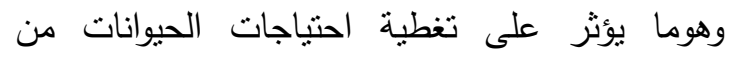

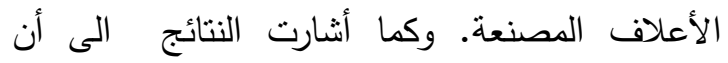

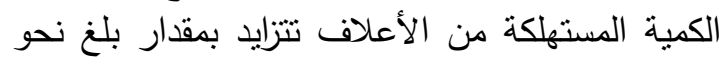

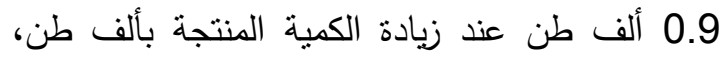

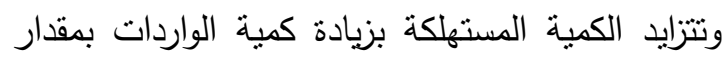
بلغ نحو 1.62 ألف طن وتتتاقص كمية الاستهلاك

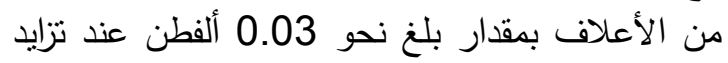

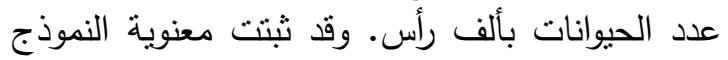
حيث أن 98\% من التغيرات التي تحدث في الكية

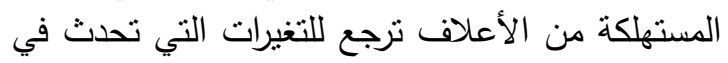
العوامل المستقلة محل الدراسة. جدول 1. الاتتاج والاستهلاك وكمية الواردات

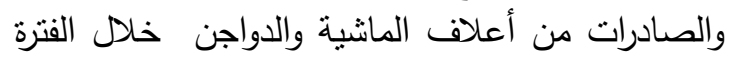
(2017-2000)

\begin{tabular}{|c|c|c|c|c|}
\hline الصادرات & الواردات & |الاستهلاك & الانتاج & |السنوات \\
\hline 2.525 & 47.35 & 2053.3 & 2008.5 & 2000 \\
\hline 3.66 & 69.8 & 2312.1 & 246 & 2001 \\
\hline 1.39 & 24.9 & 1794.5 & 1771 & 2002 \\
\hline 0.139 & 27.53 & 1532.7 & 1505.3 & 2003 \\
\hline 1.74 & 25.3 & 1809.6 & 1786 & 2004 \\
\hline 0.96 & 13.14 & 1349.7 & 1337.5 & 2005 \\
\hline 1.58 & 19.4 & 1167.6 & 1149.8 & 2006 \\
\hline 1.23 & 21.811 & 123 & 1219 & 2007 \\
\hline 3.2 & 2.65 & .9 & 1235.43 & 2008 \\
\hline 16.4 & 9 & & 1183 & 2009 \\
\hline 10.3 & 60.5 & & 8.2 & 2010 \\
\hline 4.55 & 147.5 & .0 & 0 & 2011 \\
\hline 4.6 & 173.3 & .0 & 1296.3 & 2012 \\
\hline 2.49 & 107.14 & .5 & 1199.8 & 2013 \\
\hline 1.82 & 119.6 & 123 & 121 & 2014 \\
\hline 1.78 & 134.6 & .8 & 1098 & 2015 \\
\hline 1.07 & 121.3 & 1165.2 & 1045 & 2017 \\
\hline 3.5 & 65.8 & 1483.4 & 1421.0 & المتوسط \\
\hline
\end{tabular}


جدول 2. نطور بعض المتغيرات في النموذج الآني للعلف المصنع للماشية والدواجن خلال الفترة (2000(2017

\begin{tabular}{|c|c|c|c|c|c|}
\hline سعر الصرف & عدد الحيوانات & سعر تصدير & سعر استيراد علف ماثية ودواجن & |سعر العلف & السنوات \\
\hline 3.472 & 14945 & 1482.6 & 2721.8 & 614.3 & 2000 \\
\hline 3.973 & 15630 & 1371.3 & 2228.1 & 642.9 & 2001 \\
\hline 4.500 & 16543 & 1775.5 & 4105.7 & 578.1 & 2002 \\
\hline 5.851 & 16890 & 1661.9 & 3311.9 & 792.3 & 2003 \\
\hline 6.196 & 17265 & 1912.6 & 2834.5 & 919.1 & 2004 \\
\hline 5.779 & 17547 & 3428.1 & 5913.9 & 1214.7 & 2005 \\
\hline 5.733 & 17726 & 1547.5 & 3802.7 & 1583.4 & 2006 \\
\hline 5.635 & 19053 & 1730.9 & 4560.6 & 1800.1 & 2007 \\
\hline 5.433 & 19159 & 1235.9 & 5592.5 & 1521.6 & 2008 \\
\hline 5.545 & 18232 & 13236.5 & 1016.2 & 1878.5 & 2009 \\
\hline 5.622 & 18363 & 5978.3 & 3525.7 & 1998 & 2010 \\
\hline 5.933 & 18015 & 2284.6 & 4278 & 2156.3 & 2011 \\
\hline 6.056 & 18989 & 2259.8 & 3641.1 & 2886.9 & 2012 \\
\hline 6.870 & 18530 & 6255.4 & 5470.6 & 2602.3 & 2013 \\
\hline 7.078 & 18558 & 6196.15 & 5528.3 & 3046.75 & 2014 \\
\hline 7.691 & 18247 & 6476.8 & 6048.08 & 3301.1 & 2015 \\
\hline 10.025 & 18422 & 6657.45 & 6567.86 & 3555.45 & 2017 \\
\hline 6.0 & 17771.4 & 3852.4 & 4185.1 & 1828.9 & 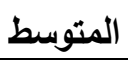 \\
\hline
\end{tabular}

المصدر: الجهاز المركزي للتعبئة العامة والاحصاء - نشرة تطور حركة الانتاج والتجارة الخارجية والمثاح للانتهلاك من أهم السلع الصناعية - اعداد متفرقة.

القريب، وعليه فإن التغيرات الفجائية تؤدى الى عدم دقة

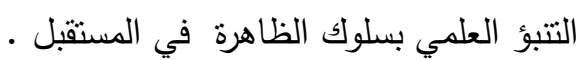

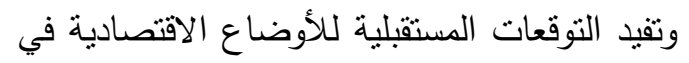

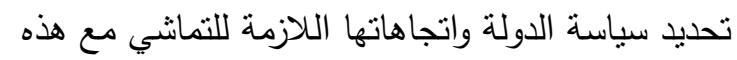

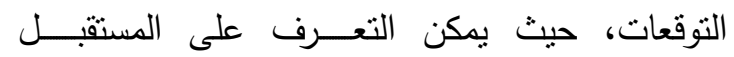

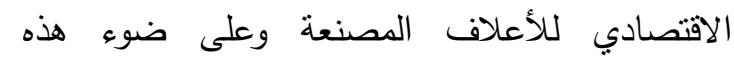

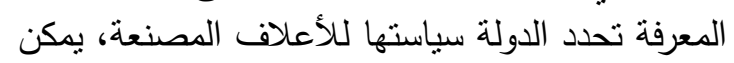

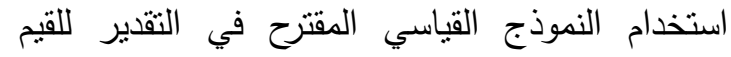
المتوقعة للمتغيرات الداخلية التي يتضمنها النموذج النيات
التببؤ بسلوك متغيرات النموذج للأعلاف المصنعة:

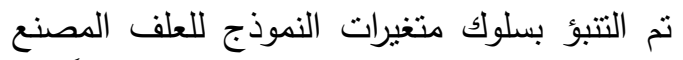

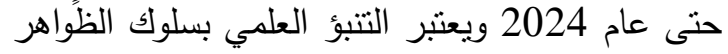

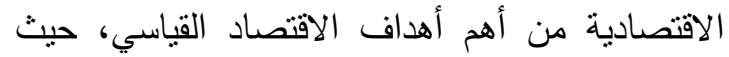

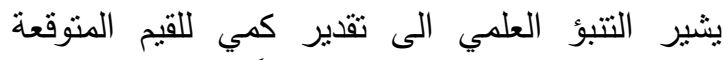

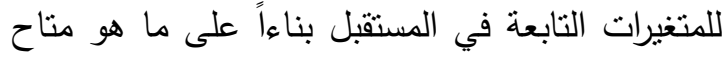

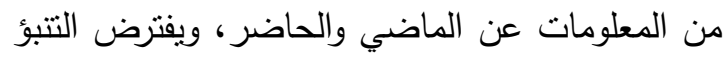

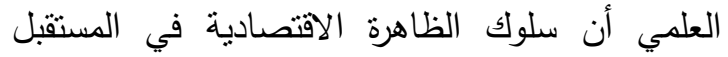

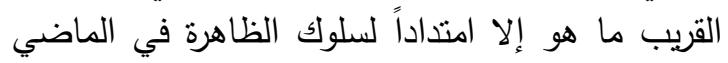


نتائج النماذج الآنية في التنبؤ بالقيم المستقبلية للواردات من العلف المصنع أفادت نتائج تقدير النموذج أن القيمة المقدرة للواردات من الأعلاف المصنعة خلال الفنرة (2019 الفير

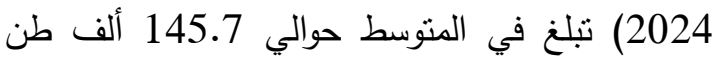

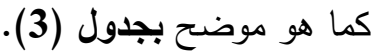

نتائج النماذج الآنية في التنبؤ بالقيم المستقبلية

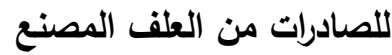
أفادت نتائج تقلير النموذج أن القيمة المقدرة للصادرات من الذرة الثامية خلال الفترة (2019 الفئ

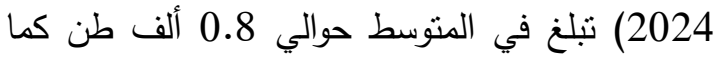
هو موضح بجدول (3) نال
نتائج النماذج الآنية في التنبؤ بالقيم المستقبلية للإنتاج من العلف المصنع أفادت نتائج تقدير النموذج أن القيمة المقدرة للإنتاج من الأعلاف المصنعة خلال الفترة (2019-2024) تبلغ في المتوسط حوالي 899.9 ألف طن كما هو النو موضح بجدول (3).

نتائج النماذج الآنية في التنبؤ بالقيم المستقبلية لكلاستهلاك من العلف المصنع أفادت نتائج تقدير النموذج أن القيمة المقدرة

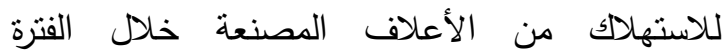

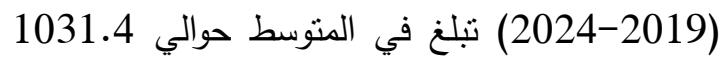

ألف طن كما هو موضح بجدول (3).

جدول 3. التتبؤ بمتغيرات الدراسة للنموذج الآني للعلف المصنع في مصر خلال الفترة (2019-2024)

\begin{tabular}{|c|c|c|c|c|c|c|}
\hline \multicolumn{4}{|c|}{ الاستهلاك } & \multicolumn{2}{|c|}{ انتاج } & \multirow{2}{*}{ 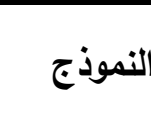 } \\
\hline \multicolumn{4}{|c|}{ ARIMA $(0,1,2)$} & \multicolumn{2}{|c|}{ ARIMA $(0,1,2)$} & \\
\hline |الحد الأدنى & الحد الأعلى & قيمة التتبوئ & الحد الأدنى & الحد الأعلى & قيمة التتبؤ & ل لسنوات \\
\hline 1575.8 & 618.9 & 1097.4 & 1436.9 & 565.9 & 1001.4 & 2019 \\
\hline 1620.1 & 587.9 & 1104.0 & 1427.2 & 526.1 & 976.6 & 2020 \\
\hline 1614.9 & 507.3 & 1061.1 & 1414.1 & 442.1 & 928.1 & 2021 \\
\hline 1607.3 & 429.1 & 1018.2 & 1398.6 & 360.6 & 879.6 & 2022 \\
\hline 1597.7 & 352.9 & 975.3 & 1381.2 & 281.0 & 831.1 & 2023 \\
\hline 1586.4 & 278.4 & 932.4 & 1362.0 & 203.0 & 782.5 & 2024 \\
\hline 1600.3 & 462.4 & 1031.4 & 1403.3 & 396.4 & 899.9 & المتوسط \\
\hline \multicolumn{3}{|c|}{ الصادرات } & \multicolumn{3}{|c|}{ الوردات } & \multirow{2}{*}{ النموذج } \\
\hline \multicolumn{3}{|c|}{ Model Random walk } & \multicolumn{3}{|c|}{ ARIMA $(0,1,2)$} & \\
\hline |الحد الأدنى & الحد الأعلى & الحد الأدنى & الحد الأعلى & الدـ الأدنى & العد الأعلى & \\
\hline 9.9 & -8.0 & 1.0 & 192.3 & 78.0 & 135.2 & 2019 \\
\hline 13.6 & -11.8 & 0.9 & 211.2 & 66.3 & 138.7 & 2020 \\
\hline 16.3 & -14.7 & 0.8 & 219.9 & 68.8 & 144.4 & 2021 \\
\hline 18.6 & -17.2 & 0.7 & 228.5 & 71.5 & 150.0 & 2022 \\
\hline 20.7 & -19.4 & 0.6 & 236.9 & 74.2 & 155.6 & 2023 \\
\hline 22.5 & -21.4 & 0.5 & 245.3 & 77.1 & 161.2 & 2024 \\
\hline 16.9 & -15.4 & 0.8 & 222.4 & 72.7 & 147.5 & المتوسط \\
\hline
\end{tabular}

stat graphic المصدر : حسبت من ننائج النموذج الآني وباستخام برنامج 
السعيد عبد الحميد البسيوني 2003. العلاقات

$$
\text { وفي ضوء ذلك توصى الدراسة بما يلى }
$$

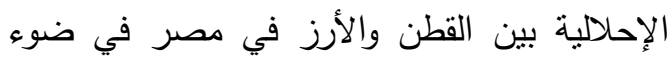

دوال استجابة العرض لكل منهما، المجلة المصرية الإنية

للاقتصاد الزراعي، المجلد 13(1)، 112 112-118.

1- الاهتمام بمحصول الذرة الثامية وبصفة خاصة

توكل يونس رذق، محمد شكري رياض، محمد حامد

العجرودى 2000.

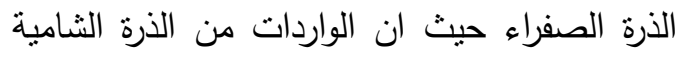

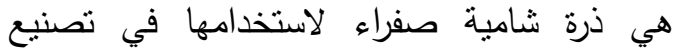

والمراعي، قسم المحاصيل، كلية الزراعة، جامعة

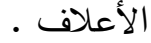

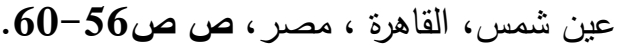

محمد احمد سعيد 2012. دراسة اقتصادية لكفاءة

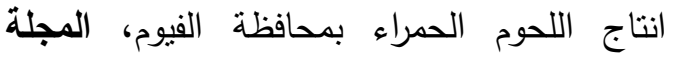

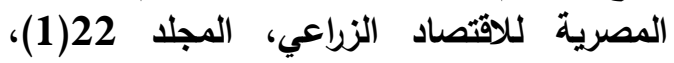

.128-122

محمد على محمد شطا 2014. التحليل الاقتصادي

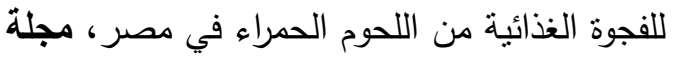

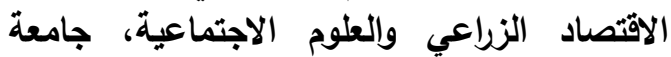

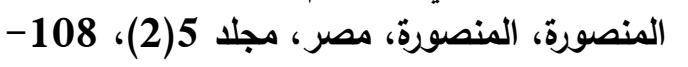

.112

2-تحديد سعر ضمان للحصول الذرة الثامية وخاصة

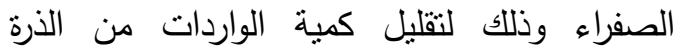

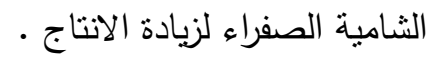

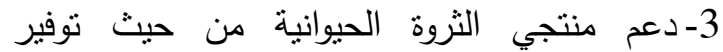

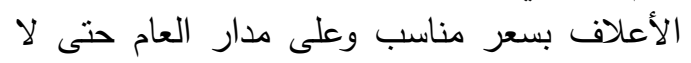

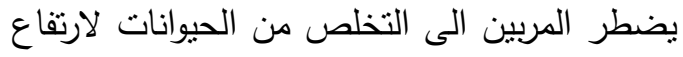

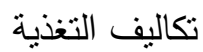

4- ضرورة الاستفادة من الفائض من الأفلغ الفاف

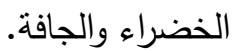

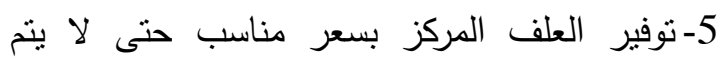
استخدام الأعلاف الخضراء بكميات كبيرة لتعويض لافرئ

النقص في الأعلاف المركزة، وبالتالي النهوض ل وزارة الزراعة واستصلاح الأراضي 2013. قطاع

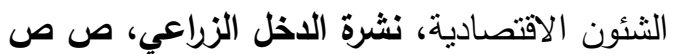

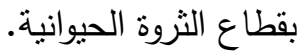




\title{
PREDICTING THE BEHAVIOR OF THE MOST IMPORTANT ECONOMIC VARIABLES FOR CATTLE AND POULTRY FEED
}

\author{
Salem ${ }^{\star}$ M.S., Thanaa E.A. Seleem and Eman F.A. Kadous \\ Agric. Economics Dept., Fac. of Agric., Ain Shams Univ., P.O. Box 68, Hadayek Shobra11241, \\ Cairo, Egypt \\ *Corresponding author: engmahmoudsalah8@gmail.com \\ Received 22 April, $2019 \quad$ Accepted 10 June, 2019
}

[107]

\section{ABSTRACT}

The traditional feed industry is based on concentrated raw feed materials and may be rich in energy or protein. The feed is a homogeneous mix of raw feed materials with some mineral salts and may be supplemented by some food additives such as vitamins, antibiotics, urea, antioxidants etc. In a soft or treated with steam and molasses and pressed into cubes, cylinders, granules or other forms. In view of the State's attention to livestock, in addition to increasing the demand for animal products for higher living standards and increased awareness of food, the feed industry is expected to rise in the next few years.

The problem of the study is limited to the impact of the agricultural production market in Egypt on many local and international economic variables, which causes fluctuations in the supply and demand of these products in the local market and foreign markets. Egypt suffers from a deficit in its trade balance of agricultural inputs. In this deficit,
\end{abstract}

as well as the decrease in the amount of concentrated fodder, mainly maize and the quantity produced from processed fodder, which also depends mainly on maize, especially poultry feed, which forms yellow maize $75 \%$ of the ingredients and therefore. The burden on the trade balance increases, especially as the dollar rises. Therefore, the study aimed to identify the economic indicators of the processed animal feed and poultry in Egypt.

The study is based on the use of descriptive statistical methods (statistical measures) and quantification of averages and percentages as well as the work of the model of the variables studied and forecasting. The current model consists of four equations. The first is the formula of local production of processed feeds. Processed, the third equation is the formula of imports of processed feeds, the fourth equation is processed feed exports.

Keywords: Processed Feeds; Animal Feed; Poultry Feed; Simultaneous Model 